\title{
Importance Sampling Estimator of Outage Probability under Generalized Selection Combining Model
}

\author{
Nadhir Ben Rached*, Zdravko Botev ${ }^{\dagger}$, Abla Kammoun*, Mohamed-Slim Alouini*, and Raul Tempone* \\ *King Abdullah University of Science and Technology (KAUST), Thuwal, Makkah Province, Saudi Arabia, \\ Email: \{nadhir.benrached, abla.kammoun, slim.alouini, raul.tempone\} @kaust.edu.sa \\ ${ }^{\dagger}$ University of New South Wales (UNSW), Sydney, NSW, Australia, \\ Email: botev@unsw.edu.au
}

\begin{abstract}
We consider the problem of evaluating outage probability (OP) values of generalized selection combining diversity receivers over fading channels. This is equivalent to computing the cumulative distribution function (CDF) of the sum of order statistics. Generally, closed-form expressions of the CDF of order statistics are unavailable for many practical distributions. Moreover, the naive Monte Carlo method requires a substantial computational effort when the probability of interest is sufficiently small. In the region of small OP values, we propose instead an efficient, yet universal, importance sampling (IS) estimator that yields a reliable estimate of the CDF with small computing cost. The main feature of the proposed IS estimator is that it has bounded relative error under a certain assumption that is shown to hold for most of the challenging distributions. Moreover, an improvement of this estimator is proposed for the Pareto and the Weibull cases. Finally, the efficiency of the proposed estimators are investigated through various numerical experiments.
\end{abstract}

Index Terms-Outage probability, generalized selection combining, order statistics, Monte Carlo, importance sampling.

\section{INTRODUCTION}

Order statistics play an important role in the performance analysis of wireless communication systems over fading channels [1]. For instance, in the generalized selection combining (GSC) model combined with maximum ratio combining (MRC) diversity technique, the output signal-to-noise-ratio (SNR) is expressed as the partial sum of ordered channel gains, i.e. squares of the amplitudes of the fading channels. More specifically, this scheme selects and combines the $L$ largest SNRs among a total of $N$ diversity branches [2]. Therefore, it is of major practical interest to evaluate the cumulative distribution function (CDF) of the sum of ordered random variables (RVs) as it can serve to compute outage probability (OP) values of GSC diversity receivers combined with MRC.

Closed-form expressions of the CDF of the partial sum of order RVs exist only for particular distributions. In [3], a unified moment generating function approach has been derived to determine the joint statistics of partial sums of ordered RVs and in particular closed-form expressions have been presented for the exponential RV. A further work on the joint statistics of partial sum of ordered exponential RVs, that is useful for instance for the analysis of OP of GSC receivers subject to self-interference, has been developed in [4]. Based on an equivalent methodology to [3], closed-form results on partial sums of ordered Gamma variates have been developed in [5] which in particular applies to OP computation at the output of
GSC combined with MRC receivers over the Nakagami fading channel. Further order statistics results in the Nakagami fading model are in [2], [6].

In the general case and apart from the exponential and Gamma RVs, closed-form expressions of the CDF of partial sums of ordered RVs are out of reach for many challenging distributions and still constitute open problems. This is for instance the case of the Log-normal RV which models shadowing [7] and weak-to-moderate turbulence channels in free space optical communication systems [8]. The Weibull variate, which has also received an increasing interest and has been shown to fit realistic propagation channels [9], is another example where the CDF of sums of order statistics is not known to admit a closed-form expression.

The use of naive Monte Carlo (MC) method can constitute a good alternative to estimate the CDF of partial sums of ordered RVs. However, since for typical wireless communication systems, more attention is accorded to small OP values, i.e. left-tail of the CDF of the sum of ordered RVs, naive MC method is known to require a substantial amount of samples to yield an accurate estimate of the left-tail of the CDF. This motivates our work where we propose a universal importance sampling (IS) estimator that yields a very precise estimate of the CDF with small computing cost [10]. We show that this estimator possesses the bounded relative error property, a relevant criterion in the context of rare event simulation, under a mild assumption that is shown to hold for many challenging distributions. A non exhaustive list includes for instance the Generalized Gamma (and in particular the Gamma and the Weibull distributions), and the $\kappa-\mu$ distributions (which includes the Rice distribution as a particular case). While this universal estimator has the feature of being applicable to a wide range of distributions, its efficiency can be significantly improved for a particular choice of distribution. This statement is validated by proposing two improvements for two particular scenarios: the Pareto and the Weibull distributions. Due to page limitation, we are not including in the current version all details. However, all proofs are available in our extended journal version [11] . Moreover, another approach has been developed in [11] and is compared against the current approach.

The rest of the paper is organized as follows. In Section II, we describe the problem setting and define the main concepts. The universal IS estimator is presented in Section III. In the same section, we present an improvement of this estimator for 
the Pareto and the Weibull scenarios. Finally, some selected numerical results are shown in Section IV to compare the performances of the proposed estimators.

\section{Problem Setting}

We consider a sequence of i.i.d RVs $X_{1}, X_{2}, \cdots, X_{N}$ with common probability density function (PDF) $f(\cdot)$. Our objective is to propose efficient MC methods to evaluate the following quantity

$$
\ell=P\left(\sum_{k=1}^{L} X^{(k)} \leq \gamma_{t h}\right),
$$

where $\gamma_{t h}$ is the threshold value, $X^{(k)}$ represents the $k^{t h}$ order statistic such that $X^{(1)} \geq X^{(2)} \geq \cdots \geq X^{(N)}$, and $L$ is an integer satisfying $1 \leq L \leq N$. The above expression of $\ell$ is a useful metric in the performance analysis of wireless communication systems, operating over fading channels. We consider transmissions between a single-antenna transmitter and an $N$-antennas receiver. Then, the quantity $\sum_{k=1}^{L} X^{(k)}$ corresponds to the total SNR when the receiver selects the $L$ best individual SNR reaching each of the diversity branches. Therefore, the quantity $\ell$ corresponds to the OP at the output of GSC combined with MRC receivers.

Unfortunately, a closed-form expression of $\ell$ is generally out of reach for many challenging distributions including, for instance, the Log-normal and the Generalized Gamma. Moreover, for small values of $\ell$, naive MC simulations is not practical since it requires a substantial number of simulations to ensure a precise estimate. Alternatively, IS techniques can deliver a reliable estimate of $\ell$ with fewer number of runs compared to naive MC simulations. Before delving into the core of our paper, it is important to define some performance metrics that serve to measure the efficiency of an unbiased estimator [10], [12]. Let $\hat{\ell}$ be an estimator of $\ell$ with $\mathbb{E}[\hat{\ell}]=\ell$, we say that $\hat{\ell}$ has bounded relative error when

$$
\limsup _{\gamma_{t h} \rightarrow 0} \frac{\operatorname{var}[\hat{\ell}]}{\ell^{2}}<\infty .
$$

Such a property implies that the number of samples needed to achieve a given accuracy remains bounded regardless of how small $\ell$ is. A stronger criterion is the asymptotically vanishing relative error property:

$$
\limsup _{\gamma_{t h} \rightarrow 0} \frac{\operatorname{var}[\hat{\ell}]}{\ell^{2}}=0 .
$$

When this criterion holds, the number of simulation runs to meet an accuracy requirement gets smaller as $\ell$ decreases.

\section{IMPORTANCE SAMPLING ESTIMATOR}

Let $\mathbf{X}=\left(X_{1}, \cdots, X_{N}\right)^{\prime}$ and $S=\left\{\boldsymbol{x}=\left(x_{1}, \cdots, x_{N}\right)^{\prime}\right.$ : $\left.\sum_{k=1}^{L} x^{(k)} \leq \gamma_{t h}\right\}$ and consider another set $S_{1}$ that includes $S$ with the assumption that $P\left(\mathbf{X} \in S_{1}\right)$ is known in closed form. Then, the probability $\ell$ is re-written as

$$
\ell=P(\mathbf{X} \in S)=P\left(\mathbf{X} \in S_{1}\right) P\left(\mathbf{X} \in S \mid \mathbf{X} \in S_{1}\right) .
$$

Hence, we express the rare event probability $\ell$ as the product of a known approximate term $P\left(\mathbf{X} \in S_{1}\right)$ and a non-rare event probability $P\left(\mathbf{X} \in S \mid \mathbf{X} \in S_{1}\right)$ that can be efficiently estimated through naive $\mathrm{MC}$ simulations. More specifically, from the above expression, we may write $\ell$ as

$$
\ell=\mathbb{E}_{g}\left[\ell_{1} \mathbf{1}_{(\mathbf{X} \in S)}\right] \triangleq \mathbb{E}_{g}\left[\hat{\ell}_{I S}\right]
$$

where $g(\cdot)$ is the PDF under which $\mathbf{X}$ is distributed according to its original PDF truncated over $S_{1}, \ell_{1}$ is equal to $P\left(\mathbf{X} \in S_{1}\right)$, and $1_{(\cdot)}$ is the indicator function. Therefore, $\hat{\ell}_{I S}$ is an importance sampling estimator with biasing PDF $g(\cdot)$.

Now, we discuss how the set $S_{1}$ is selected in order to achieve a substantial amount of variance reduction. Intuitively, the set $S_{1}$ has to be selected such that $\ell_{1}$ is close to $\ell$. In fact, the variance of $\hat{\ell}_{I S}$ is given by

$$
\operatorname{var}_{g}\left[\hat{\ell}_{I S}\right]=\ell_{1} \ell-\ell^{2}
$$

Thus, we clearly point out that the closer $\ell_{1}$ to $\ell$, the smaller the variance of $\hat{\ell}_{I S}$ is, and hence the more efficient is the estimator $\hat{\ell}_{I S}$. In particular, the estimator $\hat{\ell}_{I S}$ has bounded relative error when $\ell_{1} / \ell$ is asymptotically bounded as $\gamma_{t h}$ goes to 0 , and has asymptotically vanishing relative error in the case where $\ell_{1} / \ell$ approaches 1 as $\gamma_{t h}$ goes to 0 .

In the next subsection, we propose the simplest choice of $S_{1}$ that has the feature of being applicable to any distribution and prove that the bounded relative error holds under a mild assumption that holds for most of the challenging distributions.

\section{A. Universal IS Estimator}

The simplest choice of the set $S_{1}$ is as follows

$$
S_{1}=\left\{\boldsymbol{x}=\left(x_{1}, \cdots, x_{N}\right)^{\prime}: x^{(1)} \leq \gamma_{t h}\right\} .
$$

The probability $\ell_{1}$ is therefore given by

$$
\ell_{1}=\left(P\left(X_{1} \leq \gamma_{t h}\right)\right)^{N}
$$

The efficiency of this IS estimator is given in the following proposition

Proposition 1. For distributions satisfying $P\left(X_{1}<\gamma_{t h}\right) / P\left(X_{1} \leq \gamma_{t h} / L\right)=\mathcal{O}(1)$ as $\gamma_{t h} \rightarrow 0$, we have

$$
\limsup _{\gamma_{t h} \rightarrow 0} \frac{\ell_{1}}{\ell}<\infty
$$

Hence, the bounded relative error property holds.

Proof. The proof is given in details in [11].

The assumption $P\left(X_{1}<\gamma_{t h}\right) / P\left(X_{1} \leq \gamma_{t h} / L\right)=\mathcal{O}(1)$ is not restrictive since it is satisfied by many challenging distributions such that the Generalized Gamma (which includes in particular the Gamma and the Weibull distributions), the Rice, and the $\kappa-\mu$ distributions, see [13]. Moreover, in the independent and not identically distributed scenario, the bounded relative error property holds when the assumption of Propostion 1 is satisfied for each $X_{i}, i=1, \cdots, N$. In particular when $L=N$, this IS estimator, with the assumption in Propostion 1, is the first to achieve the bounded relative error 
property in the independent and not identically distributed case since, to the best of the authors' knowledge, this property has been only achieved in the i.i.d setting [14].

Despite its general scope of applicability, the efficiency of this universal IS estimator can be further improved if we settle for a particular distribution. This is the aim of the two following subsections where we propose other choices of $S_{1}$ in the Pareto and Weibull cases that improve the efficiency of the universal IS estimator.

\section{B. Pareto Case}

1) The Approach: The PDF $f(\cdot)$ of $X_{i}, i=1, \cdots, N$, is given as

$$
f(x)=\alpha(1+x)^{-(1+\alpha)}, x \geq 0,
$$

with $\alpha>0$. It is easy to observe that if we define $Y_{i}=$ $\alpha \log \left(1+X_{i}\right), i=1, \cdots, N$, then $Y_{i}$ has an exponential distribution with mean 1 . Using this transformation, $\ell$ is rewritten as follows

$$
\ell=P\left(\sum_{k=1}^{L} \exp \left(Y^{(k)} / \alpha\right) \leq \gamma_{t h}+L\right)
$$

Now, we will take advantage of the convexity of the exponential function to construct the set $S_{1}$. Let $\lambda_{i}>0$, $i=1,2, \cdots, L$, such that $\sum_{i=1}^{L} \lambda_{i}=1$, then we get

$$
\begin{aligned}
& \sum_{k=1}^{L} \lambda_{k} \exp \left(Y^{(k)} / \alpha-\log \left(\lambda_{k}\right)\right) \\
& \geq \exp \left(\sum_{k=1}^{L} \lambda_{k}\left(Y^{(k)} / \alpha-\log \left(\lambda_{k}\right)\right)\right) .
\end{aligned}
$$

Hence, the set $S_{1}$ is selected as

$$
\begin{aligned}
S_{1} & =\left\{\boldsymbol{y}=\left(y_{1}, \cdots, y_{N}\right)^{\prime}: \sum_{k=1}^{L} \lambda_{k} y^{(k)}\right. \\
& \left.\leq \alpha\left(\log \left(\gamma_{t h}+L\right)+\sum_{k=1}^{L} \lambda_{k} \log \left(\lambda_{k}\right)\right)\right\} .
\end{aligned}
$$

We focus now in finding a closed-form expression of $\ell_{1}$. By denoting $\gamma_{1}=\alpha\left(\log \left(\gamma_{t h}+L\right)+\sum_{k=1}^{L} \lambda_{k} \log \left(\lambda_{k}\right)\right)$ and exploiting the following representation of the order statistics $Y^{(1)}, \cdots, Y^{(L)}$, see [10]

$$
Y^{(k)}=\sum_{j=1}^{N-k+1} \frac{Z_{j}}{N-j+1},
$$

where $Z_{1}, \cdots, Z_{N}$ are i.i.d exponential RVs with mean 1 , it follows that $\ell_{1}$ is given by

$$
\ell_{1}=P\left(\sum_{i=1}^{N} \beta_{i} Z_{i} \leq \gamma_{1}\right)
$$

where

$$
\beta_{i}= \begin{cases}\sum_{j=1}^{L} \lambda_{j} /(N-i+1) & i=1=1, \cdots, N-L+1, \\ \sum_{j=1}^{N+1-i} \lambda_{j} /(N-i+1) & i=N-L+2, \cdots, N .\end{cases}
$$

Hence, $\ell_{1}$ is simply the CDF of the sum of independent exponential RVs. More specifically, a closed-form expression of $\ell_{1}$ is as follows, see [15],

$$
\ell_{1}=1-(1,0, \cdots, 0) \exp \left(\gamma_{1} \mathbf{A}\right)(1,1, \cdots, 1)^{\prime},
$$

with $\exp \left(\gamma_{1} \mathbf{A}\right)$ being the matrix exponential of $\gamma_{1} A$ and

$$
\mathbf{A}=\left(\begin{array}{ccccc}
-1 / \beta_{1} & 1 / \beta_{1} & 0 & \ldots & 0 \\
0 & -1 / \beta_{2} & 1 / \beta_{2} & \ldots & 0 \\
\vdots & \vdots & \ddots & \ddots & \vdots \\
0 & \cdots & 0 & -1 / \beta_{N-1} & 1 / \beta_{N-1} \\
0 & \cdots & 0 & 0 & -1 / \beta_{N}
\end{array}\right)
$$

2) Efficiency: We investigate in this part the efficiency of the proposed IS scheme. The main result is in the following proposition.

Proposition 2. Let $\lambda_{k}=1 / L$ for all $k \in\{1, \cdots, L\}$. Then, we have

$$
\limsup _{\gamma_{t h} \rightarrow 0} \frac{\ell_{1}}{\ell}<\infty
$$

Thus, the bounded relative error property holds.

Proof. The proof is given in details in [11].

\section{Weibull Case}

1) The Approach: we consider the case where $X_{1}, \cdots, X_{N}$ are i.i.d Weibull variates with PDF

$$
f(x)=\frac{\alpha}{\eta}\left(\frac{x}{\eta}\right)^{\alpha-1} \exp \left(-\left(\frac{x}{\eta}\right)^{\alpha}\right), x>0,
$$

where $\eta$ is the scale parameter, $\alpha$ is the shape parameter which is assumed, in this part, to satisfy $0<\alpha<1$. Consider now the RVs $Y_{i}=\left(X_{i} / \eta\right)^{\alpha}, i=1, \cdots, N$. Then, it easy to show that $Y_{i}, i=1, \cdots, N$ are i.i.d exponential RVs with mean 1 . Hence, $\ell$ is re-expressed as

$$
\ell=P\left(\sum_{k=1}^{L}\left(Y^{(k)}\right)^{1 / \alpha} \leq \gamma_{t h} / \eta\right)
$$

Let $\lambda_{i}>0, i=1, \cdots, L$, such that $\sum_{i=1}^{L} \lambda_{i}=1$. Then, using the convexity of $y \rightarrow y^{1 / \alpha}$ for $0<\alpha<1$, we get

$$
\begin{aligned}
& \left\{\sum_{k=1}^{L} \lambda_{k}\left(Y^{(k)} / \lambda_{k}^{\alpha}\right)^{1 / \alpha} \leq \gamma_{t h} / \eta\right\} \\
& \subseteq\left\{\left(\sum_{k=1}^{L} \lambda_{k}^{1-\alpha} Y^{(k)}\right)^{1 / \alpha} \leq \gamma_{t h} / \eta\right\} .
\end{aligned}
$$

Therefore, $S_{1}$ is selected as

$$
S_{1}=\left\{\mathbf{y}=\left(y_{1}, \cdots, y_{N}\right)^{\prime}: \sum_{k=1}^{L} \lambda_{k}^{1-\alpha} Y^{(k)} \leq\left(\gamma_{t h} / \eta\right)^{\alpha}\right\} \text {. }
$$

Using the same idea as in the Pareto case, the value of $\ell_{1}$ is written as

$$
\ell_{1}=P\left(\sum_{i=1}^{N} \nu_{i} Z_{i} \leq\left(\gamma_{t h} / \eta\right)^{\alpha}\right)
$$


with

$$
\nu_{i}= \begin{cases}\sum_{j=1}^{L} \lambda_{j}^{1-\alpha} /(N-i+1) & i=1=1, \cdots, N-L+1 \\ \sum_{j=1}^{N+1-i} \lambda_{j}^{1-\alpha} /(N-i+1) & i=N-L+2, \cdots, N .\end{cases}
$$

Thus, a closed-form formula for $\ell_{1}$ is given as

$$
\ell_{1}=1-(1,0, \cdots, 0) \exp \left(\gamma_{2} \mathbf{A}\right)(1,1, \cdots, 1)^{\prime},
$$

with $\gamma_{2}=\left(\gamma_{t h} / \eta\right)^{\alpha}$.

2) Efficiency: The main result is provided as follows:

Proposition 3. For $0<\alpha<1$ and arbitrary values of $\lambda_{k}$, $k=1, \cdots, L$, we have

$$
\limsup _{\gamma_{t h} \rightarrow 0} \frac{\ell_{1}}{\ell}<\infty .
$$

Hence, the bounded relative error property holds.

Proof. The proof is given in [11].

Note that, in contrast to the Pareto case where the bounded relative error property holds only for equal values of $\lambda_{k}, k=$ $1, \cdots, L$, the bounded relative error holds in the Weibull case for arbitrarily values of $\lambda_{k}$ satisfying $\lambda_{k}>0$ and $\sum_{k=1}^{L} \lambda_{k}=$ 1. Thus, the values of $\lambda_{k}$ may be optimized in order to achieve the largest amount of variance reduction. In other words, we may select the values of $\lambda_{k}$ that minimize the value $\ell_{1}$ and hence minimize the variance of the estimator $\hat{\ell}_{I S}$.

\section{NumERicAl RESUlTS AND CONCLUding REMARKS}

We provide in this section some selected simulations in order to validate the theoretical results and compare the efficiency of the proposed estimators. We define the relative error, i.e.. the coefficient of variation using $M$ replicants, of an estimator $\hat{\ell}$ as

$$
R E(\hat{\ell})=\frac{\sqrt{\operatorname{var}[\hat{\ell}]}}{\ell \sqrt{M}} .
$$

The simulations are performed for two cases: the Pareto, the Weibull. Note that the universal IS estimator described in section III-A is denoted by $\hat{\ell}_{I S, u}$ whereas the IS estimators presented in section III-B and III-C are denoted by $\hat{\ell}_{I S}$

\section{A. Pareto Case}

The sequence $X_{1}, \cdots, X_{N}$ are i.i.d Pareto RVs with parameter $\alpha=1$. We aim to estimate the CDF of the sum of $L=4$ first order statistics with $N=8$ and using the estimators $\hat{\ell}_{I S}$ and $\hat{\ell}_{I S, u}$. The corresponding results are given in Table I

TABLE I

CDF OF THE SUM OF ORDER STATISTICS For PARETO CASE With $N=8$, $L=4, \alpha=1$ AND $M=5 \times 10^{5}$.

\begin{tabular}{|c|c|c|c|c|}
\hline & \multicolumn{2}{|c|}{ IS estimator } & \multicolumn{2}{c|}{ Universal IS estimator } \\
\hline$\gamma_{t h}$ & $\hat{\ell}_{I S}$ & $R E\left(\hat{\ell}_{I S}\right) \%$ & $\hat{\ell}_{I S, u}$ & $R E\left(\hat{\ell}_{I S, u}\right) \%$ \\
\hline 1.5 & $2.21 \times 10^{-4}$ & $6.06 \times 10^{-2}$ & $2.19 \times 10^{-4}$ & 1.23 \\
1 & $2.06 \times 10^{-5}$ & $5.18 \times 10^{-2}$ & $2.11 \times 10^{-5}$ & 1.92 \\
0.5 & $2.13 \times 10^{-7}$ & $3.85 \times 10^{-2}$ & $2.09 \times 10^{-7}$ & 3.82 \\
0.1 & $1.29 \times 10^{-12}$ & $1.79 \times 10^{-2}$ & $1.29 \times 10^{-12}$ & 8.51 \\
\hline
\end{tabular}

Numerical results show that the quantity $R E\left(\hat{\ell}_{I S}\right)$ is decreasing as we decrease the threshold value $\gamma_{t h}$. Hence, $\hat{\ell}_{I S}$ , achieves numerically the asymptotically vanishing relative error property which is stronger than the theoretical result of bounded relative error proven in Proposition 2. Moreover, $\hat{\ell}_{I S}$ is much more efficient than $\hat{\ell}_{I S, u}$, which only achieves the bounded relative error as proved in Proposition 1, and the superior performance is improving as we decrease the threshold values. Thus, while $\hat{\ell}_{I S, u}$ has the feature of being applicable to a wide range of distributions, its efficiency can be significantly improved for a particular choice of distribution.

\section{B. Weibull Case}

The sequence $X_{1}, \cdots, X_{N}$ are i.i.d Weibull RVs with parameter $\eta$ and $\alpha$. Note that we set $\lambda_{k}=1 / L, k=1, \cdots, L$. The system parameters are $L=4, N=8, \alpha=0.5$, and $\eta=1$. The corresponding results are given in Table II. From the values of the relative error, we deduce that both estimators yield very accurate estimates of the unknown probability $\ell$. Moreover, we validate that they have bounded relative error which is in accordance with the theoretical results. Furthermore, the above results show that $\hat{\ell}_{I S}$ outperforms $\hat{\ell}_{I S, u}$.

TABLE II

CDF OF THE SUM OF ORDER STATISTICS FOR WEIBULL CASE WITH $N=8, L=4, \alpha=0.5, \eta=1$ AND $M=5 \times 10^{5}$.

\begin{tabular}{|c|c|c|c|c|}
\hline & \multicolumn{2}{|c|}{ IS estimator } & \multicolumn{2}{c|}{ Universal IS estimator } \\
\hline$\gamma_{t h}$ & $\hat{\ell}_{I S}$ & $R E\left(\hat{\ell}_{I S}\right) \%$ & $\hat{\ell}_{I S, u}$ & $R E\left(\hat{\ell}_{I S, u}\right) \%$ \\
\hline 1 & 0.0029 & $9.96 \times 10^{-2}$ & 0.0029 & 0.4 \\
0.5 & $3.37 \times 10^{-4}$ & 0.1 & $3.37 \times 10^{-4}$ & 0.49 \\
0.1 & $1.27 \times 10^{-6}$ & 0.11 & $1.27 \times 10^{-6}$ & 0.66 \\
0.05 & $9.79 \times 10^{-8}$ & 0.11 & $9.85 \times 10^{-8}$ & 0.71 \\
0.01 & $2.06 \times 10^{-10}$ & 0.11 & $2.06 \times 10^{-10}$ & 0.8 \\
0.005 & $1.38 \times 10^{-11}$ & 0.11 & $1.39 \times 10^{-11}$ & 0.81 \\
\hline
\end{tabular}

Finally, we aim to study the impact of varying $L$. In fact, we provide in Table III the results when $L=2$ while maintaining $N$ fixed. The outperformance of the estimator $\hat{\ell}_{I S}$ compared to $\hat{\ell}_{I S, u}$ is again clear. Moreover, this table shows that increasing $L$ affects negatively the performance of $\hat{\ell}_{I S}$ and $\hat{\ell}_{I S, u}$. Hence, we deduce that both IS estimators performs better when $L$ is close to 1 .

TABLE III

DF OF THE SUM OF ORDER STATISTICS FOR WEIBULL CASE WITH $N=8$, $L=2, \alpha=0.5, \eta=1$ AND $M=5 \times 10^{5}$.

\begin{tabular}{|c|c|c|c|c|}
\hline & \multicolumn{2}{|c|}{ IS estimator } & \multicolumn{2}{c|}{ Universal IS estimator } \\
\hline$\gamma_{t h}$ & $\hat{\ell}_{I S}$ & $R E\left(\hat{\ell}_{I S}\right) \%$ & $\hat{\ell}_{I S, u}$ & $R E\left(\hat{\ell}_{I S, u}\right) \%$ \\
\hline 0.355 & $3.38 \times 10^{-4}$ & $4.37 \times 10^{-2}$ & $3.37 \times 10^{-4}$ & 0.28 \\
0.07 & $1.28 \times 10^{-6}$ & $4.41 \times 10^{-2}$ & $1.28 \times 10^{-6}$ & 0.34 \\
0.0069 & $2.03 \times 10^{-10}$ & $4.42 \times 10^{-2}$ & $2.04 \times 10^{-10}$ & 0.37 \\
0.0035 & $1.44 \times 10^{-11}$ & $4.42 \times 10^{-2}$ & $1.45 \times 10^{-11}$ & 0.38 \\
\hline
\end{tabular}

\section{CONCLUSION}

We developed efficient importance sampling estimators to estimate the outage probability at the output of receivers with generalized selection combining scheme. We provided a universal importance sampling estimator and showed that it achieves the bounded relative error property for most of the well-practical distributions. Second, we showed how this approach can be improved if we settle for a particular distribution. Finally, we studied via various numerical results the performances of these estimators. 


\section{REFERENCES}

[1] H.-C. Yang and M.-S. Alouini, Order Statistics in Wireless Communications: Diversity, Adaptation, and Scheduling in MIMO and OFDM Systems, 1st ed. New York, NY, USA: Cambridge University Press, 2011.

[2] Y. Ma and C. C. Chai, "Unified error probability analysis for generalized selection combining in Nakagami fading channels," IEEE Journal on Selected Areas in Communications, vol. 18, no. 11, pp. 2198-2210, Nov. 2000.

[3] S. S. Nam, M.-S. Alouini, and H. C. Yang, "An MGF-based unified framework to determine the joint statistics of partial sums of ordered random variables," IEEE Transactions on Information Theory, vol. 56, no. 11, pp. 5655-5672, Nov. 2010.

[4] S. S. Nam, M. O. Hasna, and M.-S. Alouini, "Joint statistics of partial sums of ordered exponential variates and performance of GSC RAKE receivers over Rayleigh fading channel," IEEE Transactions on Communications, vol. 59, no. 8, pp. 2241-2253, Aug. 2011.

[5] S. S. Nam, Y. C. Ko, and M.-S. Alouini, "New closed-form results on ordered statistics of partial sums of Gamma random variables and its application to performance evaluation in the presence of Nakagami fading," IEEE Access, vol. 5, pp. 12 820-12 832, 2017.

[6] M.-S. Alouini and M. K. Simon, "Application of the Dirichlet transformation to the performance evaluation of generalized selection combining over Nakagami-m fading channels," Journal of Communications and Networks, vol. 1, no. 1, pp. 5-13, Mar. 1999.

[7] G. L. Stüber, Principles of Mobile Communication, 2nd Edition. Norwell, MA, USA: Kluwer Academic Publishers, 2001.

[8] S. M. Navidpour, M. Uysal, and M. Kavehrad, "BER performance of free-space optical transmission with spatial diversity." IEEE Transac- tions on Wireless Communications, vol. 6, no. 8, pp. 2813-2819, Aug. 2007.

[9] N. Sagias and G. Karagiannidis, "Gaussian class multivariate weibull distributions: theory and applications in fading channels," IEEE Transactions on Information Theory, vol. 51, no. 10, pp. 3608-3619, Oct. 2005.

[10] D. P. Kroese, T. Taimre, and Z. I. Botev, Handbook of Monte Carlo methods. N.J: Wiley, 2011.

[11] N. Ben Rached, Z. I. Botev, A. Kammoun, M.-S. Alouini, and R. Tempone, "On the sum of order statistics and applications to wireless communication systems performances," Submitted for journal publication, 2017. [Online]. Available: https://arxiv.org/pdf/1711.04280. pdf

[12] S. Asmussen and P. W. Glynn, Stochastic simulation : algorithms and analysis, ser. Stochastic modelling and applied probability. New York: Springer, 2007.

[13] N. Ben Rached, A. Kammoun, M.-S. Alouini, and R. Tempone, "A unified moment-based approach for the evaluation of the outage probability with noise and interference," IEEE Transactions on Wireless Communications, vol. 16, no. 2, pp. 1012-1023, Feb 2017.

[14] N. Ben Rached and A. Kammoun and M.-S. Alouini and R. Tempone, "Unified importance sampling schemes for efficient simulation of outage capacity over generalized fading channels," IEEE Journal of Selected Topics in Signal Processing, vol. 10, no. 2, pp. 376-388, Mar. 2016.

[15] Z. I. Botev, P. L'Ecuyer, G. Rubino, R. Simard, and B. Tuffin, "Static network reliability estimation via generalized splitting," INFORMS J. on Computing, vol. 25, no. 1, pp. 56-71, Jan. 2013. 